\title{
Software Process Simulation Frameworks in Support of Packaging and Transferring Empirical Evidence
}

\author{
Dietmar Pfahl
}

Empirical research is essential for developing a theory of software development, and - subsequently - for transforming the art of software development into engineering. In particular the latter point deals with providing evidence on the efficiency and effectiveness of tools and techniques in different application contexts. An application context is defined, firstly, by organizational aspects such as process organization, resource allocation, developer team size and quality, management rules, etc., and secondly, by the set of all other tools and techniques applied in a development project.

Controlled experiments and case studies are expensive in terms of effort and time consumption. Support for making decisions on which experiments and case studies to spend effort and time would be helpful. Such support could be provided by a software process simulation framework that is adaptable to various process configurations and accepts local efficiency and effectiveness values of tools and techniques as inputs. Simulation can generate estimates for the impact on overall project performance.

Inspired by the idea of frameworks in software development, adaptable software process simulation systems can be constructed from reusable components. The scope of a reusable component comprises construction, verification, and validation activities, and associated input/output products and resources. In order to capture the main dimensions of project performance, i.e., project duration (time), project effort (cost), and end product quality, each component has three dimensions which represent recurring patterns in software process models: functional view (activities), resource view (people, techniques, tools), and quality view (products).

Besides their cost-effectiveness due to reusability, software process simulation frameworks yield the following benefits:

- Focused improvement of techniques and tools, and associated conduct of controlled experiments and case studies, thus accelerating the generation of interesting new empirical evidence about the efficiency and effectiveness of development techniques and tools.

- Standardized representation and packaging of empirical evidence about local effectiveness and efficiency of techniques and tools in varying contexts, facilitating the systematic exploration of the impact on (global) project performance at low cost.

- Improved knowledge transfer, education, and training through visualization of the impact of local effects on global performance. 